\title{
FLAVONOIDES E OUTROS COMPOSTOS ISOLADOS DE Mimosa artemisiana Heringer E Paula\#
}

Ildomar Alves do Nascimento*

Departamento de Química, Instituto de Ciências Exatas, Universidade Federal Rural do Rio de Janeiro, BR 465 km 7, $23890-000$

Seropédica - RJ, Brasil

Raimundo Braz-Filho"\#

Laboratório de Ciências Químicas, Centro de Ciências Tecnológicas, Universidade Estadual do Norte Fluminense, Av. Alberto Lamego, 2000, 28015-620 Campos dos Goytacazes - RJ, Brasil

Mário Geraldo de Carvalho

Núcleo de Pesquisa em Produtos Naturais, Centro de Ciências da Saúde, Bloco H, $1^{\circ}$ andar, Universidade Federal do Rio de Janeiro, 21941-902Rio de Janeiro - RJ / Departamento de Química, Instituto de Ciências Exatas, Universidade Federal Rural do Rio de Janeiro, BR 465 km 7, 23890-000 Seropédica - RJ, Brasil

\section{Leda Mathias}

Laboratório de Ciências Químicas, Centro de Ciências Tecnológicas, Universidade Estadual do Norte Fluminense, Av. Alberto Lamego, 2000, 28015-620 Campos dos Goytacazes - RJ, Brasil

Fábio de Alcântara Fonseca

Instituto Estadual de Florestas, Rodovia Prefeito Américo Gianetti, s/n, 31630-900 Belo Horizonte - MG, Brasil

Recebido em 31/5/12; aceito em 17/9/12; publicado na web em 26/10/12

\begin{abstract}
FLAVONOLIGNOIDS AND OTHER COMPOUNDS ISOLATED FROM Mimosa artemisiana Heringer e Paula. This the first phytochemical investigation of Mimosa artemisiana (Leguminosae-Mimosoideae) describing the isolation and identification of quercitrin, myricitrin, 3,5,4'-trihydroxi-6,7-dimethoxyflavone (6,7-dimethylkaepferol), flavolignans, 3-O- $\beta$-D-glucopyranosil sitosterol, lupeol, sitostenone, stigmastenone, campestenone, sitosterol, stigmasterol, campesterol, methyl indole-3-carboxilate and indole-3-carboxaldehyde in the extracts from the leaves and wood of this plant. This is the first registry of 6,7-dimethoxy,4'hydroxy-flavona and the flavonolignans in this genera. The isolation of all metabolites was made by chromatographic methods and the structures were established on the basis of IR, MS, ${ }^{1} \mathrm{H}$ and ${ }^{13} \mathrm{C}$ NMR spectra analysis, comparison with literature data and GC-MS of mixtures analysis.
\end{abstract}

Keywords: Mimosa artemisiana; flavolignoids, flavonoids.

\section{INTRODUÇÃO}

Leguminosae é uma das maiores famílias de plantas abrangendo aproximadamente 650 gêneros e 18000 espécies. Ocorrem em diversas regiões do planeta, desde áreas alagadas, desertos frios ou florestas tropicais, subtropicais ou no nível do mar até uma altitude de 7000 $\mathrm{m}$. Esta família pode ser dividida em três subfamílias: Mimosoideae, Faboideae e Caesalpinoideae. Diversos estudos relatam atividades medicinais em espécies da família Leguminosae, como atividade antitumoral, ${ }^{1}$ atividade antiviral, ${ }^{2}$ antitérmica, anti-inflamatória, ${ }^{3}$ vermífuga, antisséptica e estimulante, ${ }^{4}$ entre outras.

A espécie em estudo, Mimosa artemisiana (Leguminosae), pertence à subfamília Mimosoideae e é conhecida popularmente como jurema branca. Apresenta-se como uma árvore variando entre 12-25 m de altura, ocorrendo naturalmente nos estados da Bahia, Espírito Santo e Rio de Janeiro. ${ }^{5}$ O gênero Mimosa L. compreende aproximadamente 480 espécies distribuídas em diversas regiões do mundo, principalmente no Brasil, México, Paraguai, Uruguai e Argentina. No Brasil estima-se a ocorrência de cerca de 340 espécies do gênero. ${ }^{5,6} \mathrm{~A}$ literatura descreve alguns estudos de espécie de Mimosa, que relatam o isolamento de flavonoides, ${ }^{7,8}$ saponinas e chalconas. ${ }^{9-11}$ Algumas espécies de Mimosa L. são utilizadas na medicina popular e em rituais folclóricos. São usadas como cicatrizante em queimaduras, ${ }^{11}$ como

*e-mail: ildomar@gmail.com

\#Artigo em homenagem ao Prof. Otto R. Gottlieb (31/8/1920-19/6/2011)

\#"Pesquisador Visitante Emérito - FAPERJ/UENF/UFRRJ medicamento contra picadas de cobra e de escorpião, ${ }^{12,13}$ tratamento de dor de cabeça, insônia, diarréia e febre, ${ }^{14}$ como sedativo, febrífugo e contra úlceras. ${ }^{15}$ Algumas tribos indígenas utilizam espécies de Mimosa no preparo de bebidas alucinógenas em rituais místicos e religiosos. ${ }^{16,17}$

Este é o primeiro estudo fitoquímico da espécie $M$. artemisiana e as substâncias (3), (4) e (5) estão sendo descritas pela primeira vez neste gênero. Além disso, o presente trabalho descreve o isolamento e a identificação dos flavonoides (1-3), das flavonolignanas (4) e (5) do esteroide glicosilado (6), do triterpeno lupeol (7), dos esteroides (8-13), do metil-indol-3-carboxilato (14) e do indol-3-carboxialdeído (15) como forma de contribuição para o conhecimento sobre a química do gênero Mimosa $L$.

\section{PARTE EXPERIMENTAL}

\section{Procedimentos experimentais gerais}

Os espectros de ressonância magnética nuclear de ${ }^{1} \mathrm{He} \mathrm{e}^{13} \mathrm{C}$, uni- e bi-dimensionais, foram registrados em espectrômetro Jeol Eclipse, operando a uma frequência de $400 \mathrm{MHz}$ para ${ }^{1} \mathrm{H}$ e $100 \mathrm{MHz}$ para ${ }^{13} \mathrm{C}$ e em espectrômetro Bruker Avance III ${ }^{\mathrm{TM}}$ operando a uma freqência de $500 \mathrm{MHz}$ para ${ }^{1} \mathrm{H}$ e $125 \mathrm{MHz}$ para ${ }^{13} \mathrm{C}$. Os solventes utilizados foram $\mathrm{CDCl}_{3}, \mathrm{D}_{3} \mathrm{COD}$ e $\mathrm{DMSO}_{d 6}$, utilizando o tetrametilsilano (TMS) como referência interna. Os deslocamentos químicos $(\delta)$ foram expressos em ppm e as constantes de acoplamento $(J)$ em Hz. Os espectros de absorção na região do infravermelho foram registrados 
em espectrômetro Perkin Elmer FT-IR 1600 em pastilhas de KBr. Os espectros de massas foram obtidos no aparelho GC/EM - QP-5050 Shimadzu com ionização com impacto de elétrons a $70 \mathrm{eV}$.

Os pontos de fusão, sem correção, foram obtidos em aparelho MQAPF - 301 da Microquímica. A concentração dos extratos e frações foi efetuada sob pressão reduzida em evaporador rotativo, Buchi B-480 e Fisatom 802. Análises por cromatografia em camada delgada analítica (CCDA) foram realizadas em cromatofolhas de sílica gel $60 \mathrm{GF}_{254}, \mathrm{RP}-18 \mathrm{~F}_{254 \mathrm{~s}}$ e Celulose (Merck). As substâncias foram detectadas por irradiação com lâmpada ultravioleta a 254 e 365 nm e/ou aspersão com reveladores cromogênicos (vanilina sulfúrica, seguida de aquecimento, ou solução alcoólica de cloreto férrico hexa-hidratado, com leitura no instrumento Bel Photoonics 1105).

Os critérios de pureza adotados foram a observação de uma única mancha em (CCDA), variando-se a fase móvel. Nas separações cromatográficas em coluna (CC), foram utilizados dois tipos de sílica: sílica gel 60 G (0,063-0,200 mm; 70-230 mesh ASTM) Merck e Vetec e sílica gel $60 \mathrm{G}(0,04-0,063 \mathrm{~mm} ; 230-400$ mesh ASTM e Merck). Os fracionamentos por cromatografia de exclusão foram efetuados em gel de dextrana Sephadex LH-20 da Pharmacia Fine Chemicals.

\section{Material vegetal}

O material vegetal, folhas e galhos, de $M$. artemisiana foi coletado no município de Paracambi-RJ, Brasil. A coleta e identificação da espécie foram realizadas pelo Engenheiro Florestal F. de A. Fonseca, do Instituto Estadual de Florestas (IEF-MG).

\section{Extração e isolamento dos constituintes}

Folhas secas $(495,8 \mathrm{~g})$ de $M$. artemisiana foram submetidas à extração por maceração exaustiva com $n$-hexano e, em seguida, com metanol. Os solventes foram retirados através de destilação em evaporador rotatório. Após seco em dessecador obteveram-se os extratos $15 \mathrm{~g}$ de extrato com hexano (MAFH) e $35 \mathrm{~g}$ do extrato com metanol (MAFM). 3,5 kg de madeira (galhos) da planta após secos e moídos foram submetidas à maceração exaustiva com metanol usando o mesmo procedimento descrito acima, obtendo-se o extrato MAGM (70 g). O extrato hexânico (MAFH, $15 \mathrm{~g}$ ) foi fracionado em coluna cromatográfica de sílica gel, utilizando como fase móvel hexano puro, hexano:acetato de etila $(10,20$ e $30 \%$ de acetato de etila) e acetato de etila puro. 126 frações $(15 \mathrm{~mL}$ cada) foram recolhidas e reunidas segundo a semelhança observada na análise por cromatografia de camada fina. As frações 63-66 foram reunidas e submetidas à cromatografia em coluna com gel de sílica, utilizando como fase móvel hexano:diclorometano em ordem crescente de polaridade $(0,10,20,30,50$ e $100 \%$ de diclorometano), fornecendo 50 frações de $10 \mathrm{~mL}$. A subfração 20 forneceu um precipitado que foi separado e recristalizado em metanol. Obtiveram-se 80,0 mg de um material cristalino.

A análise dos espectros permitiu reconhecer a presença dos esteroides: sitosterol (7), estigmasterol (8) e campesterol (9). O extrato metanólico de $M$. artemisiana MAFM (35 g) foi solubilizado em metanol:água (1:1) e submetido a sucessivas extrações com clorofórmio, acetato de etila e $n$-butanol, respectivamente, obtendo-se as frações MAFMC (5,4 g), MAFMAc (12,0 g), MAFMB (5,0 g) e o resíduo MAFMR (12 g). A fração MAFMC (5,4 g) foi submetida à cromatografia em coluna com gel sílica, eluída com mistura de hexano:acetato de etila em ordem crescente de polaridade $(5,10,20$, $30,40,50$ e $100 \%$ de acetato de etila). Foram recolhidas 100 frações (20 mL cada). Após análise em cromatografia de camada fina, foram selecionadas as subfrações 57-60, 65, 66 e 85-87 para estudo. O grupo de subfrações 57-60 (40 mg) foi submetido à cromatografia em coluna com gel de sílica (técnica flash) sendo eluída com diclorometano. As frações 15-19 forneceram uma substância que foi identificada como o indol-3-carboxilato de metila (13) $(4,3 \mathrm{mg})$. A fração 66 (37 mg) foi submetida ao mesmo procedimento descrito acima e as frações 20-32 deste fracionamento forneceram o indol-3-carboxialdeído (14). A fração 65 forneceu um precipitado amarelo que após filtração forneceu as substâncias (4) e (5).

O grupo de frações 85-87 também forneceu um precipitado amarelo que foi separado por filtração e identificado como o flavonoide (3). O extrato MAFMAc (12 g) foi fracionado em coluna de gel de sílica, usando como eluente mistura de $\mathrm{CHCl}_{3}: \mathrm{CH}_{3} \mathrm{OH}$ em ordem crescente de polaridade $(0,10,20,30,40,50$ e $100 \%$ de metanol). Recolheram-se 119 frações ( $15 \mathrm{~mL}$ cada fração), que foram reunidas após análise em cromatografia de camada fina. Nas frações 46-51 foi observada a formação de um precipitado amarelo $(35,0 \mathrm{mg})$. Essa fração foi submetida à cromatografia de coluna com Sephadex LH20, eluída com metanol e foram recolhidas 36 frações ( $10 \mathrm{~mL}$ cada fração). As frações 5-6 (20 mg) forneceram um precipitado amarelo, solúvel em metanol, e revelaram teste positivo para substâncias fenólicas na reação com o reagente cloreto férrico. Estas foram reunidas e, após análise com $\mathrm{RMN}{ }^{1} \mathrm{H}$ e ${ }^{13} \mathrm{C}$, identificou-se a quercetina-3-O-raminosídeo (1).

As frações 65-71 (57 mg) também revelaram teste positivo para substâncias fenólicas com o reagente cloreto férrico e forneceram um precipitado alaranjado. Este material foi filtrado em Sephadex LH-20, eluído com metanol e recolheram-se 40 frações. As frações 5-10 forneceram um precipitado amarelo (30 mg, P.F. $\left.189-192{ }^{\circ} \mathrm{C}\right)$ que, após análise com $\mathrm{RMN}$ de ${ }^{1} \mathrm{H}$ e ${ }^{13} \mathrm{C}$ e comparação com valores da literatura, permitiu identificar este material como a miricetina-3-O-raminosídeo (2).

O extrato metanólico de galhos de $M$. artemisiana (MAGM, $70,0 \mathrm{~g}$ ) foi adsorvido em sílica para coluna (1:1). A mistura obtida foi aplicada sobre uma camada de sílica para coluna em funil de Buchner e filtrado à pressão reduzida com diclorometano, acetato de etila, acetato de etila:metanol (7:3) e metanol (100\%). As soluções obtidas com cada solvente foram concentradas com destilação à pressão reduzida e secas. As frações obtidas foram codificadas da seguinte forma: MAGD (fração em diclorometano), MAGAE (fração em acetato de etila), MAGAEM (fração em acetato de etila:metanol) e MAGMM (fração em metanol). O resíduo proveniente da filtração com diclorometano (MAGD; 6,0 g) foi submetido à cromatografia em coluna com gel de sílica, usando mistura de hexano/diclorometano $(0,10,20,30,40,50$ e $100 \%$ de diclorometano) como eluente e recolhidas 52 frações $(20 \mathrm{~mL}$ cada). As frações foram reunidas segundo a semelhança observada em cromatografia de camada fina e escolheram-se as frações MAGD-33 e MAGD-36-38 para estudo.

A fração MAGD-33 forneceu um sólido branco impuro que, após análise com espectro de $\mathrm{RMN}$ de ${ }^{1} \mathrm{H}$ e ${ }^{13} \mathrm{C}$ e espectrometria de massas acoplada à cromatografia a gás, levou à identificação da mistura dos esteroides (10), (11) e (12). A fração MAGD-36-38 foi cromatografada em coluna de gel de sílica, utilizando diclorometano como eluente e recolheram-se 87 frações. Estas foram reunidas de acordo com semelhança por análise em cromatografia de camada fina. O grupo de frações 50-57 forneceu um sólido cristalino branco (26,0 mg) cuja análise dos espectros de $\mathrm{RMN}{ }^{1} \mathrm{H}$ e ${ }^{13} \mathrm{C}$ e espectro de massas foi identificado como lupeol (15). A fração MAGAE (4,5 g) foi submetida à cromatografia em coluna de gel de sílica e eluída com mistura de hexano e acetato de etila em ordem crescente de polaridade até acetato de etila 100\%. Foram recolhidas 56 frações $(20 \mathrm{~mL}$ cada). As frações 10-15 forneceram um precipitado branco amorfo $(15 \mathrm{mg})$ que apresentou coloração azul-violácea quando revelado com Reagente de Liebermann-Buchard, cuja análise do espectro de $\mathrm{RMN}{ }^{1} \mathrm{H}$ e ${ }^{13} \mathrm{C}$ levou à identificação do glicosídeo (6). 
Quercetina-3-O-raminosídeo (quercetrina) (1)

Sólido amarelo. IV $v_{\max }\left(\mathrm{cm}^{-1}\right)(\mathrm{KBr}): 3460,31 ; 2926,45 ; 1655,10$; 1608,$49 ; 1360,46 ; 1197,75 ; 1058,47$ e 1125,87 . RMN ${ }^{1} \mathrm{H}(500 \mathrm{MHz}$, $\left.\mathrm{CD}_{3} \mathrm{OD}\right) \delta_{\mathrm{H}}$ (mult.; $J$ em Hz; H) $6,19(d ; 1,8 ; \mathrm{H}-6), 6,35(d ; 1,8 ; \mathrm{H}-8)$, 7,32 ( $d ; 2,0 ; \mathrm{H}-2$ ') , 6,89 ( $d$; 8,3; H-5'), 7,29 ( $d d ; 8,3 ; 2,0 ;$ H-6'), 5,33 (d; 1,2; H-1"), 4,20 ( $d d ; 3,3$ e 1,2; H-2"), 3,73 ( $d d ; 9,5 ; 3,3 ; \mathrm{H}-3$ "), 3,31 ( $t$; 9,5; H-4"), 3,40 (dq; 9,5; 6,1; H-5"), 0,92 (d; 6,1; H-6"). $\mathrm{RMN}^{13} \mathrm{C}\left(125 \mathrm{MHz}, \mathrm{CD}_{3} \mathrm{OD}\right) \delta_{\mathrm{C}}: 159,45$ (C-2), 136,38 (C-3), 179,80 (C-4), 163,38 (C-5), 166,23 (C-7), 158,71 (C-9), 106,01 (C-10), 123,13 (C-1'), 146,59 (C-3'), 149,97 (C-4'), 100,2 (C-6), 94,90 (C-8), 117,08 (C-2'), 116,52 (C-5'), 123,00 (C-6'), 103,71 (C-1'”), 72,06 (C-2”), 72,28 (C-3”), 73,42 (C-4”), 72,18 (C-5”), 17,60 (C-6”).

Miricetina-3-O-raminosídeo (miricetrina) (2)

Sólido amarelo. IV $v_{\max }\left(\mathrm{cm}^{-1}\right)(\mathrm{KBr}): 3431,75 ; 2923,45 ; 1651,37$; 1615,$94 ; 1383,71 ; 1202,89 ; 1166,42$ e 1091,19 . RMN ${ }^{1} \mathrm{H}(500 \mathrm{MHz}$, $\left.\mathrm{CD}_{3} \mathrm{OD}\right) \delta_{\mathrm{H}}$ (mult.; $J$ em Hz; H) $6,18(d ; 2,1 ; \mathrm{H}-6), 6,35(d ; 2,1 ; \mathrm{H}-8)$, $6,93(s ; \mathrm{H}-2$ '), 6,39 ( $s$; H-6'), 5,30 (d; 1,4; H-1'), 4,20 (dd; 3,2 e 1,4; H-2"), 3,76 (dd; 9,5 e 3,2; H-3"), 3,32 ( $t$; 9,5; H-4"), 3,50 (dq; $9,5$ e 6,$2 ; \mathrm{H}-5 "), 0,95$ (d; 6,2; H-6"). RMN ${ }^{13} \mathrm{C}\left(125 \mathrm{MHz}, \mathrm{CD}_{3} \mathrm{OD}\right)$ $\delta_{\mathrm{C}}: 159,60(\mathrm{C}-2), 136,47$ (C-3), 180,00 (C-4), 163,39 (C-5), 166,17 (C-7), 158,70 (C-9), 106,01 (C-10), 122,08 (C-1'), 147,03 (C-3', 5'), 138,00 (C-4'), 100,00 (C-6), 94,86 (C-8), 109,72 (C-2', 6'), 103,81
(C-1"), 72,05 (C-2"), 72,29 (C-3"), 73,52 (C-4"), 72,20 (C-5"), 17,83 (C-6").

Eupaletina, 3,5,4'-tri-hidroxi-6,7-dimetoxiflavona (3)

Sólido amarelo; p.f. 288-291. IV $v_{\max }\left(\mathrm{cm}^{-1}\right)(\mathrm{KBr}): 3337,59$; 2923,$45 ; 1651,42 ; 1615,94 ; 1492,18 ; 1448,61 ; 1367,60 ; 1202,89$; 1166,42 e 1063,79 . RMN ${ }^{1} \mathrm{H}\left(400 \mathrm{MHz}, \mathrm{CD}_{3} \mathrm{OD}\right) \delta_{\mathrm{H}}$ (mult.; $J$ em Hz; H) 6,6; ( $s, \mathrm{H}-8), 8,1$ ( $d$; 8,8; H-2',6'), 6,9 (d; 8,8; H-3',5') 3,8 ( $s$, $\left.\mathrm{CH}_{3} \mathrm{O}-6\right) ; 3,9\left(s, \mathrm{CH}_{3} \mathrm{O}-7\right)$. RMN ${ }^{13} \mathrm{C}\left(100 \mathrm{MHz}, \mathrm{CD}_{3} \mathrm{OD}\right) \delta_{\mathrm{C}}: 147,6$ (C-2), 135,9 (C-3), 176,2 (C-4), 151,2 (C-5), 131,2 (C-6) 158,5 (C-7), 152,2 (C-9), 104,3 (C-10), 122,1 (C-1'), 158,9 (C-4'), 90,6 (C-8), 129,6 (C-2',6'), 115,2 (C-3',5').

Flavonolignana (4)

Sólido amarelo. TOF MS-ES: 494,1172 $\left(\mathrm{M}^{+}\right), 464,1120\left(\mathrm{M}^{+}\right.$ perda $\left.\mathrm{CH}_{2} \mathrm{O}\right), 463,1048\left(\mathrm{M}^{+}-\mathrm{H}-\mathrm{CH}_{2} \mathrm{O}, 100 \%\right), 283,0301\left(\mathrm{M}^{+}-\mathrm{H}-\right.$ $\left.\mathrm{C}_{11} \mathrm{H}_{14} \mathrm{O}_{4}\right) \cdot \mathrm{RMN}{ }^{1} \mathrm{H}\left(400 \mathrm{MHz}\right.$, DMSO- $\left.d_{6}\right)$ : Tabela 1. RMN ${ }^{13} \mathrm{C}(100$ MHz, DMSO- $d_{6}$ ): Tabela 1.

\section{Flavonolignana (5)}

Sólido amarelo. TOF MS-ES: 495,1274 [M+1]++; 465,1169 (perda de $\mathrm{CH}_{2} \mathrm{O}, 100 \%$ ) e 301,0469 (perda de $\mathrm{C}_{11} \mathrm{H}_{14} \mathrm{O}_{3}$ ). $\mathrm{RMN}{ }^{1} \mathrm{H}(400 \mathrm{MHz}$, DMSO- $\left.d_{6}\right)$ : Tabela $2 . \mathrm{RMN}{ }^{13} \mathrm{C}\left(100 \mathrm{MHz}, \mathrm{DMSO}-d_{6}\right)$ : Tabela 2.

Tabela 1. Dados de RMN ${ }^{1} \mathrm{H}(400 \mathrm{MHz})$ e ${ }^{13} \mathrm{C}(100 \mathrm{MHz})$ referentes à substância (4) obtidos em DMSO- $d_{6}$, inclusive resultados de HSQC e HMBC*

\begin{tabular}{|c|c|c|c|c|c|c|}
\hline \multirow{2}{*}{$\mathbf{C}$} & \multicolumn{2}{|c|}{ HMQC } & \multicolumn{2}{|c|}{ НMBC } & \multirow{2}{*}{$\begin{array}{c}\begin{array}{c}\mathrm{Lit}^{24-27} \\
\left(\mathrm{DMSO}-d_{6}\right)\end{array} \\
\delta_{\mathrm{C}}\end{array}$} & \multirow{2}{*}{$\begin{array}{c}\begin{array}{c}\mathrm{Lit}^{24-27} \\
\left(\mathrm{DMSO}-d_{6}\right)\end{array} \\
\delta_{\mathrm{H}}\end{array}$} \\
\hline & $\delta_{\mathrm{C}}$ & $\delta_{\mathrm{H}}$ & ${ }^{2} J_{\mathrm{CH}}$ & ${ }^{3} J_{\mathrm{CH}}$ & & \\
\hline 2 & 162,01 & - & $\mathrm{H}-3$ & H-2'; H-6' & 164,3 & \\
\hline 4 & 182,32 & - & & & 181,8 & \\
\hline 5 & 163,47 & - & HO-5 & & 162,9 & \\
\hline 7 & 165,05 & - & & & 161,4 & \\
\hline 9 & 157,95 & - & $\mathrm{H}-8$ & & 157,3 & \\
\hline 10 & 104,31 & - & & H-3; HO-5; H-6; H-8 & 103,8 & \\
\hline 1 ' & 124,3 & - & & H-3; H-5 & 123,7 & \\
\hline $3^{\prime}$ & 144,25 & - & & H-5 & 143,7 & \\
\hline $4^{\prime}$ & 147,76 & - & & H-2'; H-6' & 147,1 & \\
\hline $1 "$ & 126,80 & - & H-7", & $\mathrm{H}-5 "$ & 126,9 & \\
\hline $3 "$ & 148,57 & - & $\mathrm{H}-2 "$ & & 147,6 & \\
\hline $4 "$ & 136,60 & - & & H-2"; H-6" & 136,1 & \\
\hline $5 "$ & 148,57 & & H-6" & & 147,6 & \\
\hline \multicolumn{7}{|l|}{ CH } \\
\hline 3 & 104,51 & $6,87(s)$ & & & 103,9 & 6,95 \\
\hline 6 & 99,55 & $6,20(s l)$ & & HO- $5 ; \mathrm{H}-8$ & 98,9 & 6,16 \\
\hline 8 & 94,70 & $6,52(s l)$ & & H-6 & 94,1 & 6,49 \\
\hline $2^{\prime}$ & 115,40 & $7,67(s l)$ & & H-6 & 115,1 & 7,31 \\
\hline 5 & 112,42 & $7,08(d ; J=8,8 \mathrm{~Hz})$ & & & 111,0 & - \\
\hline $6^{\prime}$ & 120,47 & $7,60(d l ; J=8,8 \mathrm{~Hz})$ & & $\mathrm{H}-2$ & 120,1 & 7,26 \\
\hline $2 "$ & 106,05 & $6,75(s)$ & & & 105,4 & 6,73 \\
\hline $6 "$ & 106,05 & $6,75(s)$ & & H-2"; H-7" & 105,4 & 6,73 \\
\hline 7" & 77,26 & $5,02(d ; J=7,6 \mathrm{~Hz})$ & & & 76,2 & 4,93 \\
\hline $8 "$ & 78,59 & $4,29(m)$ & H-7” & & 78,5 & 4,25 \\
\hline \multicolumn{7}{|l|}{$\mathrm{CH}_{2}$} \\
\hline $9 "$ & 60,67 & $3,59(d ; J=11,7 \mathrm{~Hz})$ & & & 60,0 & $3,36-3,61$ \\
\hline \multicolumn{7}{|l|}{$\mathrm{MeO}$} \\
\hline $3 ", 5 "$ & 56,72 & $3,72(s)$ & & & 56,1 & 3,76 \\
\hline HO-5 & & $12,91(s)$ & & & & 12,89 \\
\hline
\end{tabular}

*O número de hidrogênios ligados a átomos de carbono foi deduzido pela análise comparativa dos espectros $\left\{{ }^{1} \mathrm{H}\right\}--$ e DEPT- ${ }^{13} \mathrm{C}$ RMN ou APT. Os deslocamentos químicos e constantes de acoplamento $(J)$ foram obtidos em $1 \mathrm{D}$ espectros de ${ }^{1} \mathrm{H}$ RMN. As sobreposições de sinais de ${ }^{1} \mathrm{H}$ foram descritas sem multiplicidade e os deslocamentos químicos aproximados deduzidos por espectros $2 \mathrm{D}$ HMQC, HMBC e ${ }^{1} \mathrm{H}-{ }^{1} \mathrm{H}-\mathrm{COSY}$. 
Tabela 2. Dados de RMN ${ }^{1} \mathrm{H}(400 \mathrm{MHz}) \mathrm{e}^{13} \mathrm{C}(100 \mathrm{MHz})$ referentes à substância (5) obtidos em DMSO- $d_{6}$, inclusive resultados de HSQC e HMBC*

\begin{tabular}{|c|c|c|c|c|c|c|}
\hline \multirow[t]{2}{*}{$\mathrm{C}$} & \multicolumn{2}{|c|}{ HMQC } & \multicolumn{2}{|c|}{ HMBC } & \multirow{2}{*}{$\begin{array}{c}\begin{array}{c}\mathrm{Lit}^{24-27} \\
\left(\mathrm{DMSO}-d_{6}\right)\end{array} \\
\delta_{\mathrm{C}} \\
\end{array}$} & \multirow{2}{*}{$\begin{array}{c}\begin{array}{c}\mathrm{Lit}^{24-27} \\
\left(\mathrm{DMSO}-d_{6}\right)\end{array} \\
\delta_{\mathrm{H}} \\
\end{array}$} \\
\hline & $\delta_{\mathrm{C}}$ & $\delta_{\mathrm{H}}$ & ${ }^{2} J_{\mathrm{CH}}$ & ${ }^{3} J_{\mathrm{CH}}$ & & \\
\hline 2 & 162,01 & - & - & H-2'; H-6' & 164,2 & - \\
\hline 3 & 136,80 & - & - & - & - & - \\
\hline 4 & 182,32 & - & & & 181,8 & - \\
\hline 5 & 163,47 & - & HO-5 & & 161,4 & - \\
\hline 7 & 165,05 & - & & & 162,9 & - \\
\hline 9 & 157,95 & - & H-8 & & 157,3 & - \\
\hline 10 & 104,31 & - & & H-3; HO-5; H-6; H-8 & 103,9 & - \\
\hline 1 ' & 124,3 & - & & $\mathrm{H}-3 ; \mathrm{H}-5$ & 123,7 & - \\
\hline 3 ' & 144,25 & - & & $\mathrm{H}-5$ & 143,7 & - \\
\hline $4^{\prime}$ & 147,76 & - & & H-2'; H-6' & 147,1 & - \\
\hline $1 "$ & 127,55 & - & H-7" & H-5", & 126,9 & - \\
\hline $3 "$ & 148,57 & - & $\mathrm{H}-2 "$ & MeO-3"; H-5" & 147,6 & - \\
\hline $4 "$ & 148,24 & - & & H-2"; MeO-4"; H-6" & 147,2 & - \\
\hline \multicolumn{7}{|l|}{$\mathbf{C H}$} \\
\hline 6 & 99,55 & $6,20(s l)$ & & HO-5; H-8 & 98,9 & 6,20 \\
\hline 8 & 94,70 & $6,52(s l)$ & & H-6 & 94,1 & 6,52 \\
\hline 2 ' & 115,40 & $7,67(s l)$ & & H-6 & 114,8 & 7,66 \\
\hline 5 & 112,42 & $7,08(d ; J=8,8 \mathrm{~Hz})$ & & & 117,0 & 7,08 \\
\hline $6{ }^{\prime}$ & 120,47 & $7,60(d l ; J=8,8 \mathrm{~Hz})$ & & $\mathrm{H}-2$ & 119,9 & 7,60 \\
\hline $2 "$ & 118,10 & $7,04(s l)$ & & & 111,7 & 7,03 \\
\hline $5 "$ & 115,95 & $6,82(d ; J=7,6 \mathrm{~Hz})$ & & & 115,3 & 6,81 \\
\hline $6 "$ & 121,18 & $6,88(d l ; J=8,8 \mathrm{~Hz})$ & & $\mathrm{H}-2 " ; \mathrm{H}-7 "$ & 120,0 & 6,87 \\
\hline $7 "$ & 76,95 & $5,02(d ; J=7,6 \mathrm{~Hz})$ & & & 76,0 & 5,01 \\
\hline $8 "$ & 78,59 & $4,29(m)$ & H-7”, & & 78,0 & 4,27 \\
\hline \multicolumn{7}{|l|}{$\mathrm{CH}_{2}$} \\
\hline $9 "$ & 60,67 & $3,59(d ; J=11,7 \mathrm{~Hz})$ & & & 60,0 & $3,37-3,58$ \\
\hline \multicolumn{7}{|l|}{ MeO } \\
\hline $3 "$ & 56,32 & $3,78(s)$ & & & 55,7 & 3,78 \\
\hline $4 "$ & 56,32 & $3,78(s)$ & & & - & - \\
\hline HO-5 & & $12,91(s)$ & & & & 12,90 \\
\hline
\end{tabular}

*O número de hidrogênios ligados a átomos de carbono foi deduzido pela análise comparativa dos espectros $\left\{{ }^{1} \mathrm{H}\right\}-$ - e DEPT- ${ }^{13} \mathrm{C}$ RMN ou APT. Os deslocamentos químicos e constantes de acoplamento $(J)$ foram obtidos em $1 \mathrm{D}$ espectros de ${ }^{1} \mathrm{H}$ RMN. As sobreposições de sinais de ${ }^{1} \mathrm{H}$ foram descritas sem multiplicidade e os deslocamentos químicos aproximados deduzidos por espectros 2D HMQC, HMBC e ${ }^{1} \mathrm{H}-{ }^{1} \mathrm{H}-\mathrm{COSY}$.

Sitosterol-3-O- $\beta$-D-glicopiranosídeo (6)

Sólido branco. IV $v_{\max }\left(\mathrm{cm}^{-1}\right)(\mathrm{KBr}): 2958,2933,2968,1462$, $1377,1367,1165$. RMN ${ }^{1} \mathrm{H}\left(400 \mathrm{MHz}, \mathrm{CD}_{3} \mathrm{OD}+\mathrm{CDCl}_{3}\right): 5,38(d ;$ H-6), 4,40 (d; 9,4; H-1'), 3,17 (dd; H-2') 3,85 (H-6'a) e 3,67 (H-6'b). $\mathrm{RMN}{ }^{13} \mathrm{C}\left(100 \mathrm{MHz}, \mathrm{CDCl}_{3}\right) \delta_{\mathrm{C}}: 140,0(\mathrm{C}-5), 36,5(\mathrm{C}-10), 42,1(\mathrm{C}-$ 13), 78,6 (C-3), 121,7 (C-6), 31,8 (C-8), 50,2 (C-9), 56,7 (C-14), 55,9 (C-17), 36,0 (C-20), 28,9 (C-24), 45,8 (C-25), 101,4 (C-1'), 73,6 (C-2') , 76,4 (C-3'), 70,3 (C-4'), 76,6 (C-5'), 61,4 (C-6'), 37,1 (C-1), 29,4 (C-2), 38,3 (C-4), 31,6 (C-7), 20,8 (C-11), 39,7 (C-12), 23,9 (C-15), 28,0 (C-16), 33,7 (C-22), 25,7 (C-23), 22, 7 (C-28), 11,1 (C18), 18,2 (C-19), 18,6 (C-21), 19,0 (C-26), 18,0 (C-27), 11,1 (C-29).

\section{Lupeol (15)}

Sólido branco; p.f. 163-165. IV $v_{\max }\left(\mathrm{cm}^{-1}\right)(\mathrm{KBr}): 3346,3066$, 2941, 2872, 1637, 1450. EM m/z 426, 189 (100\%), 218, 297, 175. RMN ${ }^{1} \mathrm{H}\left(200 \mathrm{MHz}, \mathrm{CDCl}_{3}\right) \delta_{\mathrm{H}}$ (mult.; $J$ em Hz; H) 3,1 $(d d ; 9,9$ e $5,2 ; \mathrm{H}-3), 2,3$ ( $d d d ; 10,8 ; 10,8 ; 5,3 ; \mathrm{H}-19), 4,6$ (dl; 2,2; H-29), 4,5 ( $m$; H-29). RMN ${ }^{13} \mathrm{C}\left(50 \mathrm{MHz}, \mathrm{CDCl}_{3}\right) \delta_{\mathrm{C}}: 38,8(\mathrm{C}-1), 27,3(\mathrm{C}-2)$, 78,3 (C-3), 38,8 (C-4), 55, 2 (C-5), 18, 2 (C-6), 34, 2 (C-7), 40,7 (C8), 50,3 (C-9), 37,1 (C-10), 20,8 (C-11), 25,0 (C-12), 37,9 (C-13), 42,7 (C-14), 27,9 (C-15), 35,5 (C-16), 42,9 (C-17), 48,2 (C-18), 47,3 (C-19), 150,9 (C-20), 29,7 (C-21), 39,7 (C-22), 28,6 (C-23), 15,3
(C-24), 16,0 (C-25), 15,9 (C-26), 14,5 (C-27), 17,9 (C-28), 109,3 (C-29), 19,2 (C-30).

\section{RESULTADOS E DISCUSSÃO}

A análise dos dados espectrométricos [IV, RMN ${ }^{1} \mathrm{H}$ e ${ }^{13} \mathrm{C}$ (1D e 2D)] da substância (1) permitiu atribuir a estrutura do flavonoide quercetrina para esta substância. Além da análise dos espectros IV, o espectro de RMN ${ }^{1} \mathrm{H}$ apresentou sinais dos hidrogênios $\mathrm{H}-6\left(\delta_{\mathrm{H}} 6,19\right.$; $\mathrm{d} ; J=1,8 \mathrm{~Hz})$ que acopla com o H-8 $\left(\delta_{\mathrm{H}} 6,35 ; \mathrm{d} ; J=1,8 \mathrm{~Hz}\right)$ e do H-6' $\left(\delta_{\mathrm{H}} 7,29 ; \mathrm{dd} ; J_{1}=8,3 \mathrm{~Hz} ; J_{2}=2,0 \mathrm{~Hz}\right)$ que acopla com H-5' $\left(\delta_{\mathrm{H}}\right.$ $6,89 ;$ d; $J=8,3 \mathrm{~Hz})$ e H-2' $\left(\delta_{\mathrm{H}} 7,32 ; \mathrm{d} ; J=2,0 \mathrm{~Hz}\right)$. A comparação dos dados de $\mathrm{RMN}{ }^{13} \mathrm{C}$ com valores da literatura foi compatível com a estrutura do flavonoide (1), Figura 1. ${ }^{18,19}$

Os espectros de $\mathrm{RMN}{ }^{1} \mathrm{H}$ (1D e 2D) do flavonoide (2) mostraram sinais de absorções em $\delta_{\mathrm{H}} 6,18(\mathrm{~d} ; J=2,1 \mathrm{~Hz})$ e $\delta_{\mathrm{H}} 6,35(\mathrm{~d} ; J=2,1 \mathrm{~Hz})$ referentes aos H-6 e H-8, respectivamentes, do anel A do flavonoide um sinal simples em $\delta_{\mathrm{H}} 6,93(2 \mathrm{H})$ atribuído aos H-2' e H-6' do anel B. A unidade de raminose foi sugerida baseada na presença dos sinais de hidrogênio anomérico em $\delta_{\mathrm{H}} 5,30(\mathrm{~d} ; J=1,4 \mathrm{~Hz} ; 1 \mathrm{H})$ e pelo sinal de metila em $0,95(\mathrm{~d} ; J=6,2 \mathrm{~Hz} ; 3 \mathrm{H})$. Os demais sinais da unidade do carboidrato foram confirmados pela presença de correlação no 


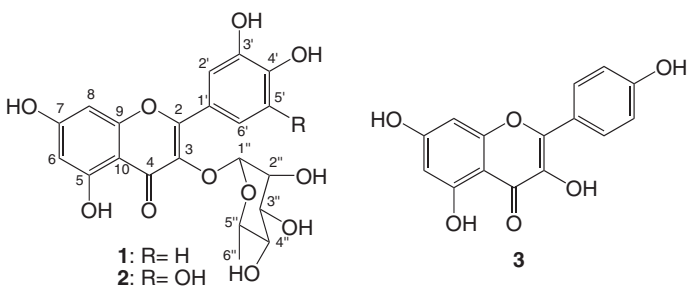

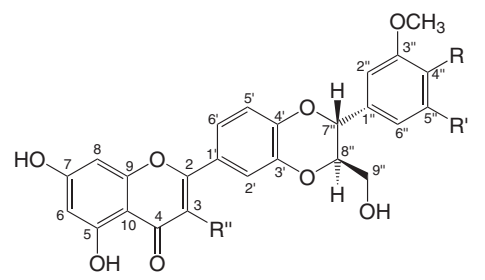

4: $\mathrm{R}=\mathrm{OH} ; \mathrm{R}^{\prime}=\mathrm{OCH}_{3} ; \mathrm{R}^{\prime \prime}=\mathrm{H}$
5: $\mathrm{R}=\mathrm{OCH}_{3} ; \mathrm{R}^{\prime}=\mathrm{H} ; \mathrm{R}^{\prime \prime}=\mathrm{OH}$

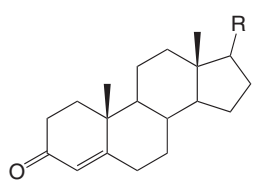

10: $R=(c)$ 11: $R=(b)$ 12: $R=(a)$
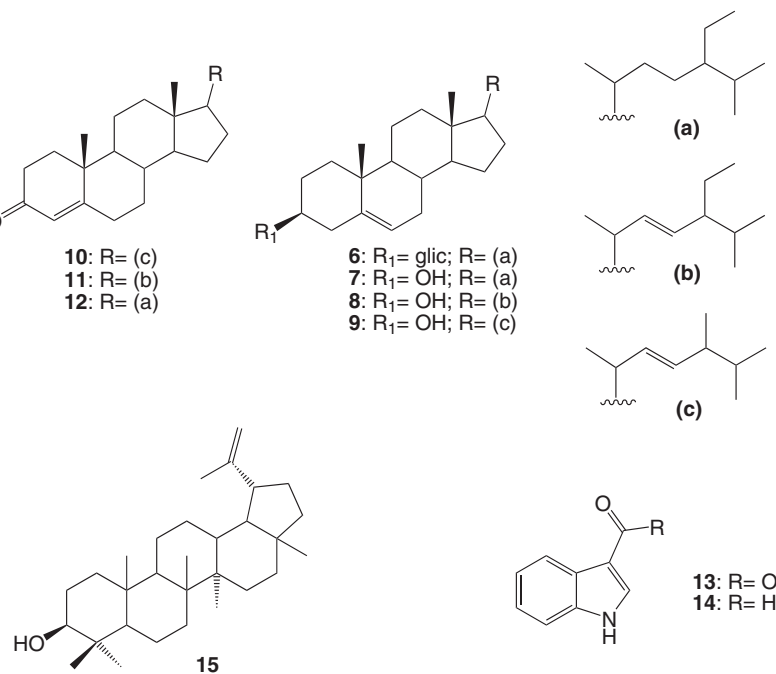

13: $\mathrm{R}=\mathrm{OCH}_{3}$
Figura 1. Substâncias isoladas de Mimosa artemisiana

espectro HMQC entre os seguintes sinais: $\delta_{\mathrm{C}} 72,0(\mathrm{C}-2$ ") $) / \delta_{\mathrm{H}} 4,2$ $\left(\mathrm{dd} ; J_{1}=1,4 \mathrm{~Hz} ; J_{2}=3,2 ; \mathrm{H}-2\right.$ ") $\left.) \delta_{\mathrm{C}} 72,2(\mathrm{C}-3 ")\right) / \delta_{\mathrm{H}} 3,7\left(\mathrm{dd} ; J_{1}=3,2\right.$ $\mathrm{Hz} ; J_{2}=9,5 \mathrm{~Hz} ; \mathrm{H}-3$ " $) ; \delta_{\mathrm{C}} 73,5(\mathrm{C}-4$ " $) / \delta_{\mathrm{H}} 3,3\left(\mathrm{t} ; \mathrm{H}-4\right.$ ”) e $\delta_{\mathrm{C}} 72,2(\mathrm{C}-$ 5 ") $/ \delta_{\mathrm{H}} 3,5$ (dq; H-5"). A análise do espectro de RMN ${ }^{13} \mathrm{C}$ permitiu identificar sinais correspondentes a carbonos não hidrogenados (11 carbonos), metínicos (três sinais representando 4 carbonos $\mathrm{sp}^{2}$ e 5 carbonos $\mathrm{sp}^{3}$ oxigenados) além um carbono metílico (grupo metila da raminose em $\delta_{\mathrm{C}} 17,8$ correlacionando com o sinal em $\delta_{\mathrm{H}} 0,95(\mathrm{~d}$; $J=6,2 \mathrm{~Hz}$ ). O sinal em 103,81 foi atribuído ao carbono anomérico C-1", que apresentou sinal de correlação com $\delta_{\mathrm{H}} 5,30(\mathrm{~d}, 1,4 \mathrm{~Hz})$ atribuído ao H-1" no espectro de HMQC. Os dados de RMN obtidos para esta substância corroboram os valores descritos na literatura para a miricetrina. ${ }^{18-20}$

A estrutura da 3,5,4'- tri-hidróxi-6,7-dimetóxiflavona (3) foi definida com base na análise dos espectros de IV e de RMN ${ }^{1} \mathrm{H}$ $(400 \mathrm{MHz})$ e ${ }^{13} \mathrm{C}(100 \mathrm{MHz})$ uni- e bidimensionais, incluindo experimento de NOESY. Além de dois sinais simples em $\delta_{\mathrm{H}} 3,85$ e 3,95 de grupos metoxilas, o espectro de $\mathrm{RMN}{ }^{1} \mathrm{H}$ apresentou três sinais de hidrogênios ligados a anel aromático; um sinal simples em $\delta_{\mathrm{H}} 6,65$ e dois dubletos em $\delta_{\mathrm{H}} 8,1(8,8 \mathrm{~Hz} ; 2 \mathrm{H})$ e $6,9(8,8 \mathrm{~Hz} ; 2 \mathrm{H})$, respectivamente, típicos de um sistema AA'BB'. A ausência de sinal de NOE na frequência dos dubletos permitiu eliminar a posição das metoxilas em 4' e 3, e localizá-las em 6 e 7 devido ao NOE no sinal em 6,65 do $\mathrm{H}-8$ proposto. Estas propostas foram confirmadas pela análise do espectro HMBC que apresentou sinais de correlação a longa distância entre hidrogênios e sinais de carbonos quaternários como o ${ }^{2} J_{\mathrm{CH}}$ de C-7/H-8; C-9/H-8 e C-10/H-8, e ${ }^{3} J_{\mathrm{CH}}$ de C-6/H-8, C-6/ $\mathrm{CH}_{3} \mathrm{O}-6$ e C-7/ $\mathrm{CH}_{3} \mathrm{O}-7$. Estas informações permitiram propor a estrutura 3,5,4'-tri-hidróxi-6,7-dimetóxiflavona, também conhecida como eupaletina (3). Os dados de $\mathrm{RMN}{ }^{1} \mathrm{H}$ e ${ }^{13} \mathrm{C}$ estão de acordo com valores registrados na literatura para esta substância. ${ }^{21-23}$

As substâncias (4) e (5) foram identificadas através de análise dos espectros de IV e RMN ${ }^{1} \mathrm{H},{ }^{13} \mathrm{C}$ (BBD e APT) uni- e bidimensionais da fração contendo ambas substâncias, incluindo comparação com dados da literatura. ${ }^{2} \mathrm{O}$ espectro na região do infravermelho de $\mathbf{4}+$ 5) apresentou bandas de absorção em $3425 \mathrm{~cm}^{-1}\left(v_{\mathrm{OH}}\right), 2922$ e 2849 $\left(v_{\mathrm{CH}}\right.$ de $\mathrm{CH}_{2}$ e $\left.\mathrm{CH}_{3}\right), 1657$ ( $v_{\mathrm{C}=\mathrm{O}}$ de carbonila conjugada), 1621 e 1436 (deformação axial $v_{\mathrm{C}=\mathrm{C}}$ de sistema aromático). $\mathrm{O}$ espectro de $\mathrm{RMN}{ }^{1} \mathrm{H}$ da substância 4 apresentou um sinal em $\delta_{\mathrm{H}}$ 6,87 característico de H-3 de flavonas. Esse sinal apresentou acoplamento a uma ligação com o sinal em $\delta_{\mathrm{C}} 104,5$ observado no espectro de HSQC. O espectro de $\mathrm{RMN}{ }^{1} \mathrm{H}$ exibiu um sistema $\mathrm{ABX}$ em $\delta_{\mathrm{H}} 7,08$ (d; $J=8,8 ; \mathrm{H}-5$ '), 7,67 (sl; H-2') e 7,60 (dl; $J=8,8$ Hz; H-6'). Esses sinais estão consistentes com um anel $\mathrm{C}$ trissubstituído de uma flavona, cujos acoplamentos foram confirmados através do espectro bidimensional ${ }^{1} \mathrm{H}-{ }^{1} \mathrm{H}$ COSY. Os sinais simples e largos em $\delta_{\mathrm{H}} 6,20$ e 6,52, referentes aos H-6 e $\mathrm{H}-8$, respectivamente, são consistentes com o anel A de um flavonoide 5,7-dioxigenado. Estes hidrogênios apresentaram sinais de correlação com carbonos em $\delta_{\mathrm{C}}$ 99,55 (C-6) e 94,70 (C-8) no espectro de HSQC, respectivamente. A análise do espectro bidimensional de correlação ${ }^{1} \mathrm{H} \mathrm{x}{ }^{1} \mathrm{H}$ COSY revelou um sistema de acoplamento que permitiu sugerir a presença dos hidrogênios metínicos e metilênicos oxigenados (H-7, H-8 e H-9a,b), sugerindo que estes hidrogênios pertencem a um fragmento de propanol que forma um anel dioxânico ligado a um anel aromático. A análise do espectro bidimensional HSQC permitiu observar que estes hidrogênios apresentaram sinais de acoplamento a uma ligação com os carbonos $\delta_{C} 77,26$ (C-7”), 78,59 (C-8") e 60,67 (C-9"), respectivamente. Estes sinais permitiram propor a presença da substância 4 na mistura, que foi confirmada pelas informações adicionais descritas a seguir. O sinal simples em $\delta_{\mathrm{H}} 3,72$ foi atribuído aos hidrogênios das metoxilas ligadas em C-3", 5" de (4).

$\mathrm{Na}$ Tabela 1 estão as atribuições de todos os sinais de ${ }^{1} \mathrm{H}$ e ${ }^{13} \mathrm{C}$ e as correlações observadas para (4). $\mathrm{O}$ espectro de massas de 4 apresentou picos em $\mathrm{m} / \mathrm{z}$ 494,1172 $\left(\mathrm{M}^{+}\right), 464,1120\left(\mathrm{M}^{+}\right.$perda de $\left.\mathrm{CH}_{2} \mathrm{O}\right), 463,1048\left(\mathrm{M}^{+}\right.$perda de $\mathrm{H}$ e de $\left.\mathrm{CH}_{2} \mathrm{O}, 100 \%\right)$ e 283,0301 $\left[\mathrm{M}^{+-}\right.$211,0970, (perda de $\mathrm{H}$ e de $\mathrm{C}_{11} \mathrm{H}_{14} \mathrm{O}_{4}$ )] confirmando a proposta da estrutura. $\mathrm{O}$ experimento NOESY 1D obtido através da irradiação em $\delta_{\mathrm{H}}$ 7,67 (H-2') forneceu nOe no sinal em $\delta_{\mathrm{H}} 6,87$ (H-3), confirmando a posição proposta para os hidrogênios referentes a esses sinais; o espectro bidimensional NOESY apresentou sinal de interação espacial entre a metoxila em $\delta_{\mathrm{H}} 3,72$ e H-2", 6" $\left(\delta_{\mathrm{H}} 6,75\right)$. O espectro de carbono-13 PENDANT apresentou, além dos sinais já atribuídos a 4, sinais adicionais em $\delta_{\mathrm{C}} 148,57$ (C-3”), 136,80 (C-3),

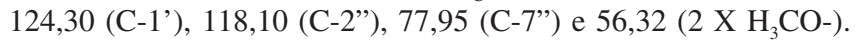
Esses sinais permitiram sugerir a presença da substância $\mathbf{5}$, conforme relacionado na Tabela 2, que apresenta os dados de $\mathrm{RMN}{ }^{1} \mathrm{H} \mathrm{e}{ }^{13} \mathrm{C}$ da mesma. Os sinais de três hidrogênios em $\delta_{\mathrm{H}} 7,04(\mathrm{sl}), 6,82$ (d; $J=$ $7,6 \mathrm{~Hz})$ e 6,88 (dl; $J=8,8 \mathrm{~Hz}$ ) foram atribuídos aos hidrogênios H-2", H-5" e H-6", respectivamentes, do anel aromático 1,3,4-trissubstituído da substância 5. Os sinais dubleto em $\delta_{\mathrm{H}} 5,02$ (H-7"; $J=7,6$ $\mathrm{Hz}$ ), multipleto em 4,29 e 3,59 observados no espectro de ${ }^{1} \mathrm{H}$ RMN e ${ }^{1} \mathrm{H}-{ }^{1} \mathrm{H}-\mathrm{COSY}$ representam os hidrogênios ligados aos carbonos $s p^{3}$ da unidade $\mathrm{C}_{6} \mathrm{C}_{3}$ proposta. Esta proposta foi confirmada pela análise do espectro HMQC ${ }^{1} \mathrm{~J}_{\mathrm{HC}}$ de 7 " $(5,02 / 76,95), 8$ " $(4,29 / 78,59)$ e 9" $(3,59 / 60,67)$. Os valores das constantes de acoplamento entre os hidrogênios 7" e 8" ( $J=7,6 \mathrm{~Hz})$ permitiram definir a configuração relativa trans no sistema dioxânico de $\mathbf{5}$. O espectro bidimensional HMBC foi útil na atribuição dos deslocamentos químicos dos demais carbonos através da identificação de sinais de acoplamentos a longa 
distância ${ }^{2} J_{\mathrm{CH}} \mathrm{e}^{3} J_{\mathrm{CH}}$, (Tabela 2). O singleto em $\delta_{\mathrm{H}} 3,78(6 \mathrm{H})$ foi atribuído aos hidrogênios das metoxilas em C-3" e C-4" de 5. A análise do espectro de NOESY 1D, cuja irradiação no sinal em 3,78 gerou nOe nos sinais de H-2" e H-5", confirmou a posição das metoxilas vizinhas ao H-2" e H-5". O espectro de massas de $\mathbf{5}$ apresentou picos em $\mathrm{m} / \mathrm{z}$ 495,1274 [M+1] ++; 465,1169 (perda de $\mathrm{CH}_{2} \mathrm{O}, 100 \%$ ) e 301,0469 (perda de 194,0942, $\mathrm{C}_{11} \mathrm{H}_{14} \mathrm{O}_{3}$ ) estando de acordo com a estrutura proposta. A análise detalhada da integração dos espectros de hidrogênio permitiu propor a percentagem relativa de $4(32,6 \%)$ e $5(67,4 \%)$ na fração analisada.

A saponina esteroidal $\mathbf{6}$ e o triterpeno $\mathbf{1 5}$ foram identificados através da análise dos espectros de IV, RMN ${ }^{1} \mathrm{H}$ e ${ }^{13} \mathrm{C}$ (APT), espectros de massas e comparação com valores da literatura para $\mathrm{o}$ glicopiranosil-sitosterol e o lupeol. ${ }^{28,29}$

Os esteroides 10, 11 e 12 tiveram suas estruturas definidas mediante análise dos espectros de IV e RMN ${ }^{1} \mathrm{H}$ e ${ }^{13} \mathrm{C}$ (APT), que apresentaram sinais compatíveis para a mistura de esteroides contendo uma enona no anel A. A análise dos espectros de massas detectados para os componentes da mistura permitiu observar os valores de $\mathrm{M}^{+}$. em $\mathrm{m} / z 398$ para 10 (TR: 21,425); m/z 410 para 11 (TR: 22,158) e m/z 412 para 12 (TR: 23,742). A comparação dos espectros com os da espectroteca confirmou as propostas. Análise semelhante foi feita para a fração contendo as substâncias sitosterol (7), estigmasterol (8) e campesterol (9). Além dos sinais compatíveis para o esqueleto esteroidal, os espectros de massas de cada componente, identificados pelos picos no cromatograma do $C G, T_{R} 21,55 ; 21,17$ e 19,84 min, apresentaram, respectivamente, $\mathrm{m} / \mathrm{z}$ em $414\left(\mathrm{M}^{+}, \mathbf{7}\right), 412\left(\mathbf{M}^{+}, \mathbf{8}\right)$ e $400\left(\mathbf{M}^{+}, \mathbf{9}\right)$. A confirmação das estruturas foi feita por comparação com dados da literatura ${ }^{30-32}$ e com o uso de padrões em cromatografia de camada analítica com mistura semelhante isolada de outras espécies vegetais.

As estruturas das substâncias $\mathbf{1 3}$ e $\mathbf{1 4}$ foram definidas com base dos espectros de IV, massas e RMN ${ }^{1} \mathrm{H} \mathrm{e}{ }^{13} \mathrm{C}$ uni- e bidimensionais. Além da comparação dos dados de RMN com valores da literatura. ${ }^{33,34}$ Os espectros de massas de ambas foram compatíveis com as propostas, sendo $m / z, 175\left(\mathrm{M}^{+}\right), 144\left(\mathrm{M}^{+\cdot}-\mathrm{OCH}_{3}\right) ; 116(144-\mathrm{C}=\mathrm{O})$; 89 (116- HCN) para 13, indol-3-carboxilato de metila, e $\mathrm{m} / z: 145$ $\left(\mathrm{M}^{+}, 80 \%\right), 144\left(\left[\mathrm{M}^{+}\right]-\mathrm{H} ; 100 \%\right), 116(144-\mathrm{C}=\mathrm{O}), 89(116-\mathrm{HCN})$ para 14, indol-3-carboxialdeído.

\section{AGRADECIMENTOS}

Ao CNPq, à CAPES e FAPERJ pelos auxílios e bolsas concedidos. Ao MSc. F. de A. Fonseca (IEF-MG) pela coleta e identificação do material vegetal.

\section{REFERÊNCIAS}

1. Muller, B. M.; Kraus, J.; Franz, G.; Planta Med. 1989, 55, 536.

2. Sydiskis, R. J.; Owen, D. G.; Lohr, J. H.; Rosler, K. H. A.; Blomster, R. N.; Antimicrob. Agents Chemother. 1991, 35, 2463.

3. Chopra, R. N.; Nayar, S. L.; Chopra, I. C.; Glossary of Indian Medicinal Plants, Council of Scientific and Industrial Research: New Delhi, 1956.

4. Mendonça, D. E.; Onofre, S. B.; Rev. Bras. Farmacogn. 2009, 19, 577.

5. Lorenzi, H.; Árvores brasileiras: manual de identificação e cultivo de plantas arbóreas nativas do Brasil, $2^{\mathrm{a}}$ ed., Ed. Plantarum: Nova Odessa, 1998, vol. 2.
6. Silva, J. S.; Sales, M. F. de; Rodriguesia 2008, 59, 435.

7. Yusuf, U. K.; Abdullah, A.; Bakar, B.; Itam, K.; Abdullah, F.; Sukari, M. A.; Biochem. Syst. Ecol. 2003, 31, 443.

8. Kirk, L. F.; Møller, M. V.; Christensen, J.; Staerk, D.; Ekpe, P.; Jaroszewski, J. W.; Biochem. Syst. Ecol. 2003, 31, 103.

9. Jiang, Y.; Berrurier, M. H.; Anton, R.; J. Nat. Prod. 1991, 54, 1247.

10. Englert, J.; Weniger, B.; Lobstein, A.; Anton, R.; J. Nat. Prod. 1995, 58, 1265 .

11. Dominguez, X. A.; Garcia, S.; Williams, H. J.; Ortiz, C.; Scott, A. I.; J. Nat. Prod. 1989, 53, 865.

12. Mahanta, M.; Mukherjee, A. K.; J. Ethnopharmacol. 2001, 75, 55.

13. Girish, K. S.; Mohanakumari, H. P.; Nagaraju, S.; Vishwanath, B. S.; Kemparaju, K.; Fitoterapia 2004, 75, 378.

14. Ngo Bum, E.; Dawack, D. L.; Schmutz, M.; Rakotonirina, A.; Rakotonirina, S. V.; Portet, C.; Jeker, A.; Olpe, H.-R.; Herrling, P.; Fitoterapia 2004, 75, 309.

15. Desmarchelier, C.; Romão, R. L.; Coussio, J.; Ciccia, G.; J. Ethnopharmacol. 1999, 67, 69.

16. Gupta, M. P.; Arias, T. D.; J. Nat. Prod. 1979, 42, 234

17. Pachter, I. J.; Zacharias, D. E.; Ribeiro, O.; J. Org. Chem. 1959, 24, 1285 .

18. Agrawal, P. K.; Carbon-13 NMR of Flavonoids, Ed. Elsevier: Amsterdam, 1989.

19. Santos, P. M. L.; Schripsema, J.; Kuster, R. M.; Rev. Bras. de Farmacogn. 2005, 15, 321.

20. Mahmoud, I. I.; Marzouk, M. S. A.; Moharram, F. A.; El-Gind, M. R.; Hassan, A. M. K.; Phytochemistry 2001, 58, 1239.

21. Wei, X.; Huang, H.; Wu, P.; Cao, H.; Ye, W.; Biochem. Syst. Ecol. 2004, 32, 1091.

22. Pandey, R.; Maurya, R.; Singh, G.; Sathiamoorthy, B.; Naik, S.; Int. Immunopharmacol. 2005, 5, 541.

23. Córdova, W. H. P.; Mesa, L. G.; Hill, A. L. P.; Lima, C. N.; Lamas, G. D.; Suárez, M. O.; Dominguez, R. S.; Pharmacologyonline 2006, 3, 757.

24. Guz, N. R.; Stermitz, F. R.; Johnson, J. B.; Beeson, T. D.; Willen, S.; Hsiang, J. F.; Lewis, K.; J. Med. Chem. 2001, 44, 261.

25. Pan, L.; Chin, Y. W.; Chai, H. B.; Ninh, T. N.; Soejarto, D. D.; Kinghorn, A. D.; Bioorg. Med. Chem. 2009, 17, 2219.

26. Guz, N. R.; Stermitz, F. R.; J. Nat. Prod. 2000, 63, 1140; Pettit, G. R.; Meng, Y.; Clare, A. S.; Doubek, D. L.; Knight, J. C.; Cichacz, Z.; Pettit, R. K.; Chapuis, J-C.; Schmidt, J. M.; J. Nat. Prod. 2003, 66, 259.

27. Harbone, J. B.; Mabry, T. J.; The Flavonoids - Advances Reserch, Chapman and Hill: London, 1976

28. Costa, H. N. R.; Santos, M. C.; Alcântara, A. F. C.; Silva, M. C.; França, R. C.; Piló-Veloso, D.; Quim. Nova 2008, 31, 744.

29. Mahato, S. B.; Kundu, A. P.; Phytochemistry 1994, 37, 1517.

30. Forgo, P.; Kövér, K. E.; Steroids 2004, 69, 43

31. Macari, P. A. T.; Emerenciano, V. P.; Ferreira, Z. M. G. S.; Quim. Nova 1990, 13, 260.

32. Kontiza, I.; Abatis, D.; Malakate, K.; Vagias, C.; Roussis, V.; Steroids 2006, 71, 177.

33. Yue, Q.; Miller, C. J.; White, J. F. Jr.; Richardson, M. D.; J. Agric. Food Chem. 2000, 48, 4687.

34. Lin, C. F.; Huang, Y. L.; Cheng, L. Y.; Sheu, S.; Chen, C. C.; Zhogyi Xиebao 2006, 17, 103. 